\title{
A New Stimulus Set for Cognitive Research
}

\author{
Luke Rosedahl \& F. Gregory Ashby \\ University of California, Santa Barbara
}

\begin{abstract}
A new stimulus set is described that could prove useful for a wide variety of different cognitive experiments. Each stimulus is a schematic representation of a fish and the stimulus ensemble could potentially be varied on many dimensions. These new stimuli have a number of attractive properties, including 1) a higher degree of ecological validity than most stimuli used in cognitive research, and 2) a large number of qualitatively different types of dimensions that can be varied. Psychophysical properties of the stimuli were studied in two experiments. Experiment 1 measured just noticeable differences on two prominent dimensions, and Experiment 2 used multidimensional scaling to study the structure of the underlying perceptual representations. The result is an ecologically valid stimulus set with a well-understood perceptual representation.
\end{abstract}

\section{Introduction}

Research on any basic cognitive process is facilitated when stimuli are used that have a well understood dimensional structure. Trying to understand a cognitive process is easiest when there is no ambiguity about perceptual processing. For this reason, many cognitive studies have used simple, artificial stimuli that vary on only a few dimensions. Included in this list are Gabor patches that vary in spatial frequency and orientation, and lines that vary in length and orientation.

Unfortunately, simplistic, low-level stimuli of this type are far removed from the natural, real-world objects and events that shaped the evolution of our cognitive systems. For this reason, another large group of cognitive studies have used more complex stimuli - such as natural images, or cartoon figures that vary on a number of binary values. One problem with these studies is that - in most cases - we have only a vague understanding of how participants perceive these stimuli, and this uncertainty makes it difficult to draw strong inferences about the cognitive processes under study.

These concerns served as motivation for this article. Specifically, our goal was to develop a set of stimuli that have more ecological validity than Gabor patches, that do not appeal to any pre-existing categories, that are easy to generate and control in a laboratory setting, and that have a known perceptual structure. Our solution was to create a set of cartoon caricatures of fish - illustrated in Figure 1 - that potentially could be varied on many different dimensions. Matlab code that generates these images is available through the Open Science Framework at osf.io/bwcvy.

The entire list of stimulus attributes that can be manipulated are shown in Table 1. Specifically, numerical values of any of these 14 constants can be set in the Matlab code that generates the stimuli. Our initial investigations varied five of these dimensions: 1) mouth angle (i.e., denoted by A1 in

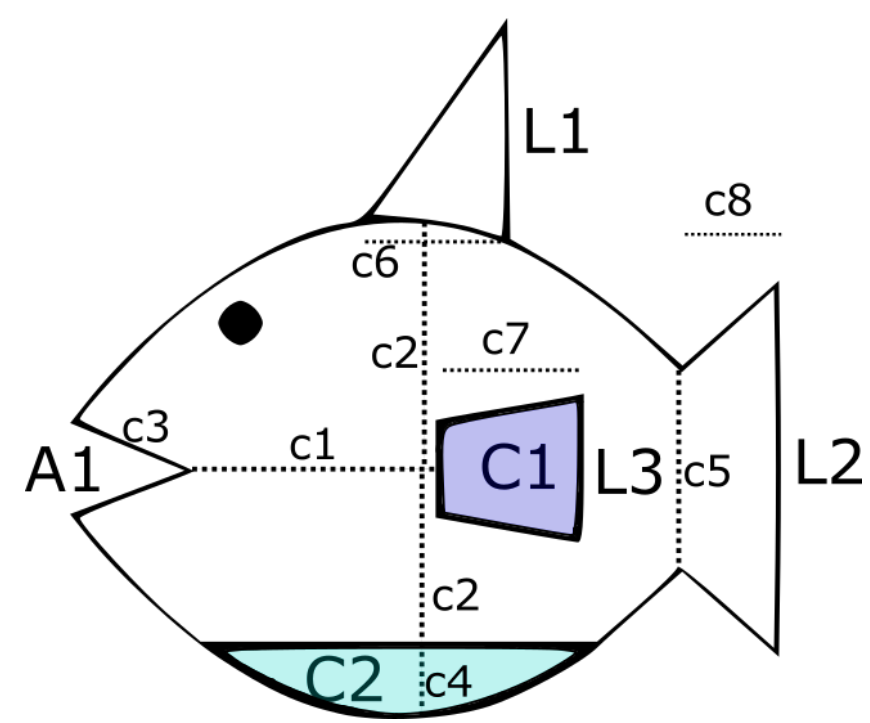

Figure 1. An example fish stimulus, along with labels on every dimension that can be manipulated.

Figure 1), 2) dorsal fin height (i.e., L1), 3) tail height (i.e., L2), 4) pectoral fin color (i.e., C1), and 5) belly color (i.e., C2).

We investigated the perceptual representations of these new stimuli in two separate experiments. Experiment 1 estimated the just noticeable difference (jnd) of mouth angle (A1) and dorsal fin height (L1). Ideally, all stimulus dimensions listed in Table 1 should be scaled in jnd units. If so, then the perceptual salience of a one unit change in one dimension would be approximately equal to the perceptual salience of a one unit change in any other dimension. We expect tail height and pectoral fin height to have similar jnds as dorsal fin height. We did not estimate jnds for any color dimensions 
because the structure of color space has been studied meticulously in many prior experiments (e.g., Jameson \& Hurvich, 1959).

In Experiment 2, participants rated the similarity of stimulus pairs that varied on five dimensions - mouth angle, dorsal fin height, tail height, belly color, and pectoral fin color. The data were then analyzed via multidimensional scaling to examine the structure of perceptual space and especially the relationship among the various perceptual dimensions.

Table 1

Manipulable Stimulus Values

\begin{tabular}{|l|l|l|l|}
\hline Item & Description & Type & Value \\
\hline A1 & Mouth Angle & Variable & NA \\
\hline L1 & Dorsal Fin height & Variable & NA \\
\hline L2 & Tail height & Variable & NA \\
\hline L3 & Pectoral Fin Height & Constant & 15 \\
\hline C1 & Pectoral Fin Color & Variable & NA \\
\hline C2 & Belly Color & Variable & NA \\
\hline c1 & Pectoral Fin Location & Constant & $(0,0)$ \\
\hline c2 & Overall Fish Height & Constant & 100 \\
\hline c3 & Mouth Length & Constant & 100 \\
\hline c4 & Belly Height & Constant & 15 \\
\hline c5 & Tail Base Height & Constant & 40 \\
\hline c6 & Dorsal Fin Width & Constant & 30 \\
\hline c7 & Pectoral Fin Width & Constant & 30 \\
\hline c8 & Tail Width & Constant & 20 \\
\hline
\end{tabular}

\section{Experiment 1}

Experiment 1 estimated jnds of mouth angle and dorsal fin height in an attempt to create units of measurement on each dimension that equate changes in perceptual salience. A QUEST staircase procedure (implemented in psychotoolbox) was used to select stimuli and to determine the final estimated JNDs. The JNDs were then used to find a mathematical function that identifies the physical units of degrees (in the case of mouth angle) and number of pixels (in the case of dorsal fin height) that correspond to any given number of jnds. These transformations allow researchers to construct stimuli that have any desired configuration in a perceptual space where the units on each dimension are jnds.

\section{Methods}

Participants and Design. Eighty participants were recruited from the University of California at Santa Barbara community. All participants were undergraduates who received course credit in return for their participation. Each session consisted of 10 blocks of 50 trials and lasted approximately 45 minutes.

Procedure. Participants were instructed to determine which of a pair of fish had a larger value along a given dimension. Participants were informed of the dimension of inter- est before each block and that they were to make judgments about that dimension on all trials during the block. Mouth angle was the target dimension for half of the 10 blocks of 50 trials and dorsal fin height was the target for other half. On each trial, one fish was presented for $500 \mathrm{~ms}$, then a white noise mask was presented for $250 \mathrm{~ms}$, and then a second fish was displayed for $500 \mathrm{~ms}$. The participant would then respond with the fish that they thought was larger along the targeted dimension by pressing either A for the first fish or B for the second fish.

Stimuli and Apparatus. On every trial, one of the two fish, randomly selected, had a base value on the relevant dimension (i.e., 20, 40, 60, 80, or 100 degrees or pixels, for mouth angle and dorsal fin height, respectively). The other fish had a dimensional value that was selected by the QUEST procedure. All other stimulus dimensions remained constant for the entire experiment and were set to values shown in Table 1. No feedback was given after any response, and the next trial started after a $250 \mathrm{~ms}$ pause. For all thresholds, data points were determined to be outliers if they fell outside three standard deviations from the median or were reported by QUEST as guessing.

\section{Results}

The results are shown in Figure 2. Each dot represents the estimated jnd for one participant. Table 2 lists the mean jnds across participants. To investigate more closely how the jnd changes with stimulus value, we performed tests for linear and quadratic trend. The linear trend was highly significant in both conditions [mouth angle: $F(1,156)=100.27, p<$ .001 ; dorsal fin height: $F(1,148)=154.81 ; p<.001]$, whereas the quadratic component of the relationship was nonsignificant in both cases [mouth angle: $F(1,156)=$ $.432 ; p=0.51$; dorsal fin height: $F(1,148)=1.374 ; p=$ 0.24]. Figure 2 also shows the best-fitting lines, which had slopes of 0.105 for mouth angle and 0.109 for dorsal fin height and intercepts of 2.8 for angle and 0.3 for fin height. Note that the slopes provide estimates of the Weber fractions for each dimension. Weber's law predicts intercepts of 0 , so the 2.8 intercept for mouth angle represents a slight violation of Weber's law, which we suspect is due to the temporal separation and noise mask between stimuli. Note that the Weber fraction for fin height matches traditional Weber fraction estimates of approximately .1 for line length (i.e., Tudusciuc \& Nieder, 2010).

Table 2

Mean jnds (across participants) for each stimulus value

\begin{tabular}{|l|l|l|l|l|l|}
\hline Dimension & 20 & 40 & 60 & 80 & 100 \\
\hline Mouth Angle & 4.9 & 7.0 & 9.1 & 11.2 & 13.3 \\
\hline Dorsal Fin Height & 2.5 & 4.7 & 6.8 & 9.0 & 11.2 \\
\hline
\end{tabular}

This new stimulus set will be most useful for cognitive 

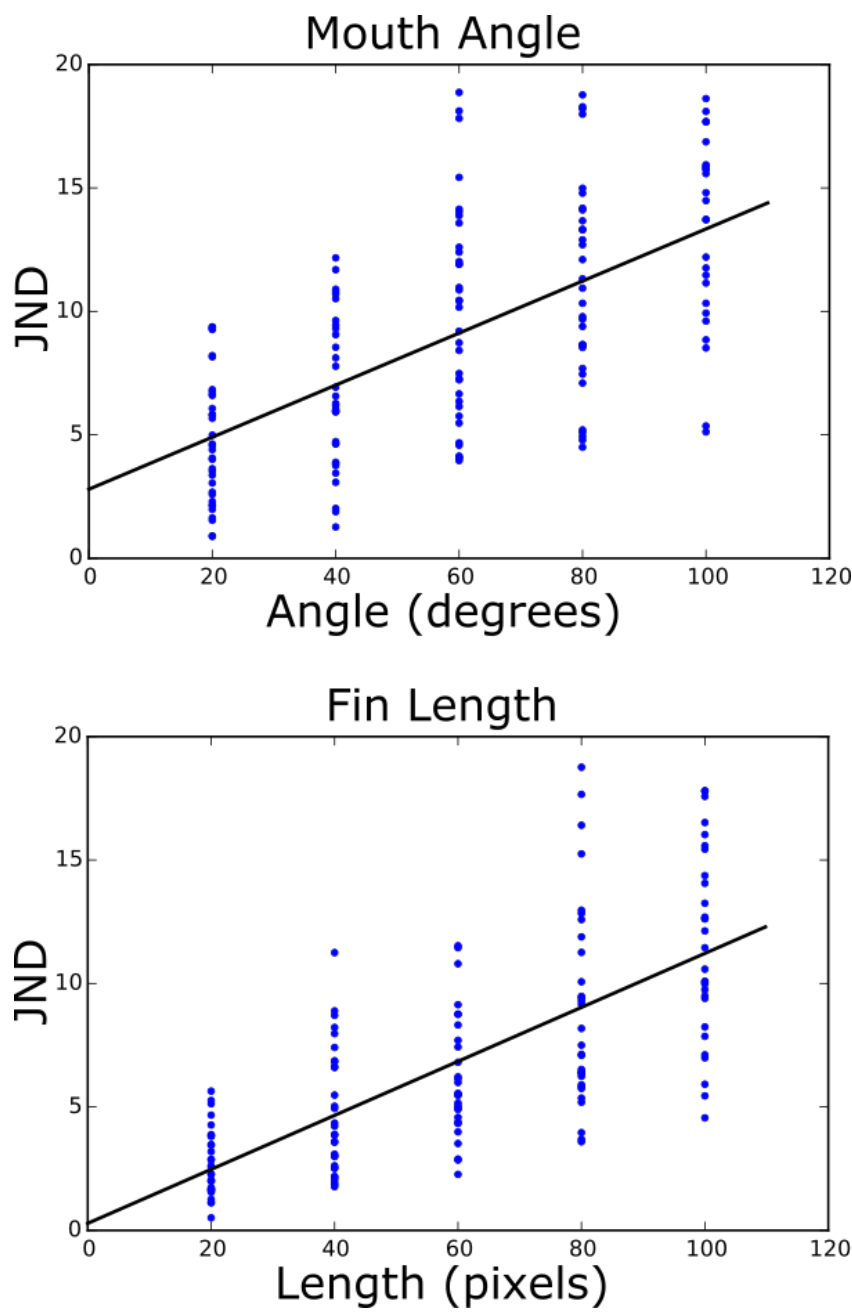

Figure 2. JNDs and the best-fitting linear regression. Each dot is the jnd for a single participant.

research if experimenters have an easy method to generate stimuli in a space where the units are jnds. For example, this would mean that the perceptual difference on each dimension between stimulus values 5 and 6 would be the same as the perceptual difference between stimulus values of say 9 and 10. But for the length and height dimensions, experimenters most naturally would work in units of pixels, whereas for the angle dimensions the natural units are degrees or radians. So we need transformations that convert a desired number of jnds to pixels and degrees.

First consider fin height. Let $X$ denote the subjective dimension where the units are jnds. The best-fitting regression line for fin height in Figure 2 was

$$
\text { jnd }=0.109 H+0.3
$$

where $H$ is fin height in pixels. For simplicity, round this to

$$
\text { jnd }=0.1 H \text {. }
$$

The subjective dimension $X$ has a fixed unit of one jnd, but the zero point is rather arbitrary. So we will arbitrarily assume that $X=0$ for a fin height of $H=10$ pixels. Given this assignment, the Appendix shows that the transformation between $X$ and $H$ is accomplished via

$$
H=10 \times 1.1^{X} .
$$

So, for example, to create 3 dorsal fin heights that are spaced 3 jnds apart (e.g., at $X=3,6$, and 9), an experimenter would create fin heights of 13, 18, 24 pixels, respectively (after rounding to the nearest pixel). We expect that this same transformation should prove equally effective for all height and width dimensions listed in Table 1.

The transformation from $X$ to degrees of mouth angle $\theta$ is similar since the slope of the regression line is also approximately 0.1 . However, in the case of mouth angle the regression intercept is large enough that it should not be ignored. So the empirical constraint we will try to preserve is

$$
\text { jnd }=0.1 \theta+2.8 \text {, }
$$

where $\theta$ is mouth angle in degrees. The Appendix shows that this more complex relationship produces the transformation

$$
\theta=38 \times 1.1^{X}-28 .
$$

So, for example, to create 3 mouth angles that are spaced 3 jnds apart (e.g., at $X=3,6$, and 9), an experimenter would create fish with mouth angles of $22.6^{\circ}, 39.3^{\circ}$, and $61.6^{\circ}$. To preserve the fish-like qualities of the stimulus, mouth angles should be less than $180^{\circ}$, which corresponds to a jnd value of $X=17.8$. Therefore, researchers should select $X$ values for mouth angle that fall in the range $[0,17]$.

To convert stimulus values from $X$ space back to a space where the dimensions are in the physical units of degrees and pixels, one can just solve Equations 3 and 5 for $H$ and $\theta$, respectively, which produces

$$
X_{H}=10.5 \ln H-24.2
$$

and

$$
X_{\theta}=10.5 \ln (\theta+28)-38.2
$$

\section{Discussion}

In Experiment 1, we estimated jnds for two of the primary dimensions of the fish stimuli: mouth angle and dorsal fin height. As predicted by Weber's law, the relationship between jnd and stimulus value was linear for both dimensions, and in both cases the slope was approximately 0.1 - a value that closely matches prior psychophysical research (e.g., Tudusciuc \& Nieder, 2010). These results were then used to identify transformations to a perceptual space in 
which the units on every dimension are jnds. For example, by using Equations 3 and 5, an experimenter could choose levels of mouth angle and any of the height or width dimensions that are an equal number of jnds apart. Note that in this case, a change from one level to the next would be equally perceptually salient on every dimension.

Creating similar transformations for the color dimensions is a much trickier process because color vision varies widely across individuals and depends critically on previous color exposure (Jameson \& Hurvich, 1959). In addition, careful pyschophysical control of color dimensions requires extensive calibration (e.g., of the monitor and ambient light). As a result, we did not vary any color dimensions in Experiment 1. For these reasons, we recommend using the color dimensions cautiously in any experiment where it is important to control the number of jnds that separate different levels on each dimension.

\section{Experiment 2}

Experiment 1 provided a method for creating perceptual dimensions in which the unit of measurement is the jnd. Experiment 2 uses multidimensional scaling (MDS) to examine the relationships among dimensions.

\section{Methods}

Participants and Design. Sixty-six participants were recruited from the UCSB community. All participants were undergraduates who received course credit in return for their participation. Each session consisted of 496 trials and lasted approximately one hour.

Procedure. For each trial, participants saw two fish side by side and provided a similarity rating on a scale from 1-7, with 1 meaning not at all similar and 7 meaning extremely similar. Five dimensions were varied: mouth angle, dorsal fin height, tail height, pectoral fin color, and belly color. Two values were selected for each dimension, resulting in 496 pairs of stimuli. Each subject saw each pair once throughout the course of the experiment.

Stimuli and Apparatus. Values of 40/80 degrees and 40/80 pixels were used for mouth angle and both fin heights respectively. For the color dimensions, two colors pairs were selected from Munsell space for each color dimension and converted to RGB values. The colors chosen and their RGB values are listed in Table 3. The fish were presented side by side on the screen and participants could take up to 3 seconds to supply a similarity rating. If they took longer, they were prompted to respond faster.

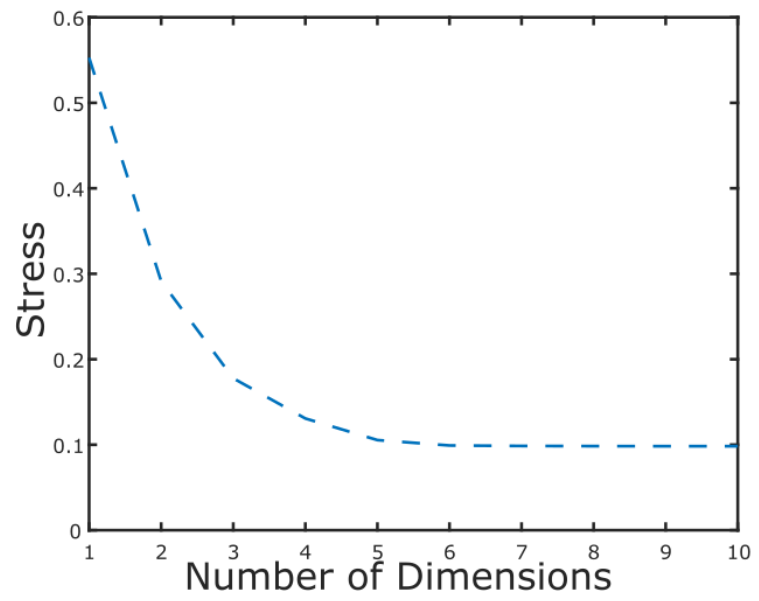

Figure 3. MDS Stress

Table 3

Fin and Belly Colors Used in Experiment 2

\begin{tabular}{|l|l|l|}
\hline Dimension & Munsell & RGB \\
\hline Fin 1 & 10 PB 8/6 & {$[.781, .769, .953]$} \\
\hline Fin 2 & 10 PB 4/6 & {$[.387, .355, .539]$} \\
\hline Belly 1 & 7.5 PB 4/6 & {$[.340, .367, .539]$} \\
\hline Belly 2 & 7.5 PB 4/14 & {$[.266, .340, .739]$} \\
\hline
\end{tabular}

All participants performed a similarity rating on every pair of fish. There were 2 values on each of 5 dimensions, which resulted in 32 different fish (i.e., $2^{5}$ ). All possible pairs were presented to each participant, except the 32 possible identical pairs, which were omitted. This resulted in a total of 496 different pairs of fish. The similarity ratings were then averaged across all participants and combined into a dissimilarity matrix for analysis. Nonmetric MDS was then done in Matlab using mdscale to minimize Kruskal's normalized stress-1 (a commonly used MDS stress measure).

\section{Results}

Stress, which measures the goodness-of-fit of the MDS solution, is plotted in Figure 3 as a function of the number of MDS dimensions. Lower stress means a better fit, and note that, as expected, stress decreases sharply up until the solution has five dimensions. Increasing the number of dimensions above five produces only negligible decreases in stress (i.e., of about 1\%). Thus, the optimal MDS solution has five dimensions, and any additional dimensions are likely just fitting noise.

An examination of stress values, as in Figure 3, is a good way to determine the optimal dimensionality, but a poor way of determining whether the best MDS solution provides a good or bad account of the data, because absolute stress values are difficult to interpret. A better way to assess the quality 


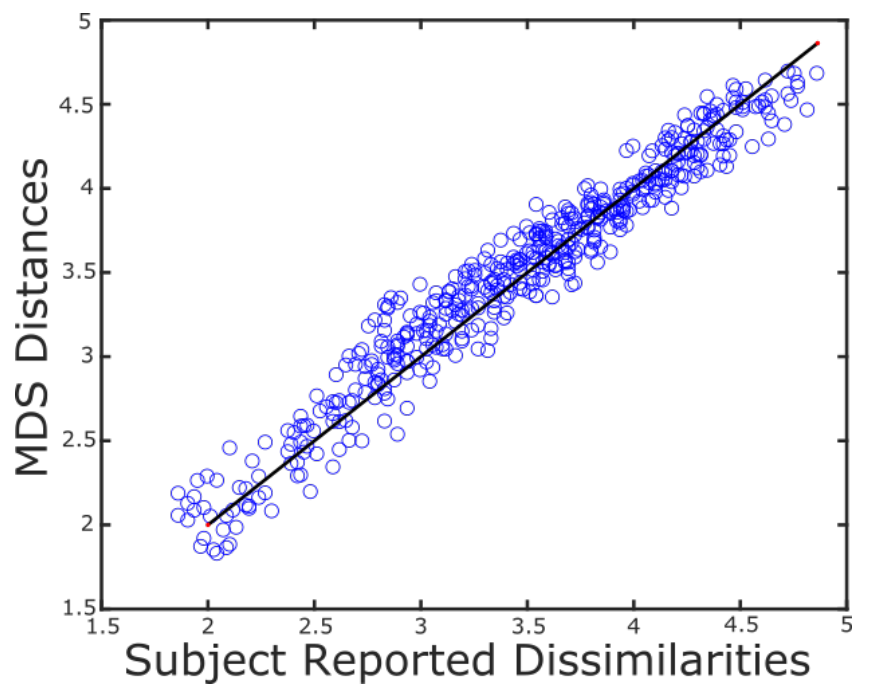

Figure 4. MDS Shepard plot for the five-dimensional MDS solution. The line has slope one and intercept zero. The Pearson correlation coefficient is .974 .

of the MDS solution is to examine the Shepard plot, which is a scatter plot with one point for each pair of stimuli that plots the distance between the stimuli in the MDS solution against their rated dissimilarity ${ }^{1}$ The Shepard plot for the five-dimensional MDS solution is shown in Figure 4. The inter-point distances in a good MDS solution should mirror the participant dissimilarity ratings, so the closer the points are to a line with slope one (and intercept zero), the better the fit. As can be seen, the data are clustered nicely around this line with a Pearson correlation coefficient of .974 , showing that the MDS solution provides a good account of the judged similarities.

The main purpose of Experiment 2 was to examine the relationships among pairs of stimulus dimensions for the fish stimuli. Consider four fish that are constructed by factorially combining two dorsal fin heights and two mouth angles. The common graphical representation of these stimuli would be as four points that form a rectangle in a two-dimensional stimulus space that has fin height as one dimension and mouth angle as the other. The main goal of Experiment 2 was to examine whether the orthogonality of this stimulus representation is preserved in the perceptual representation - that is, in the best MDS solution. Under appropriate conditions, orthogonality of perceptual dimensions signals that the stimulus dimensions are perceived independently (Ashby \& Townsend, 1986), so another way to describe the goal of Experiment 2 is that we are examining whether the different pairs of stimulus dimensions are perceived independently.

The dimensions of an MDS solution are arbitrary, as is the unit of measurement. Thus, to compare the MDS solution to the standard orthogonal stimulus representation that is used to describe any set of stimuli created by a factorial combination of binary-valued dimensions, we aligned the MDS representation to the orthogonal stimulus representation as closely as possible using a Procrustes transformation.

Procrustes transformations are shape preserving, so the only operations allowed are translation, reflection, rotation, and uniform stretching or shrinking. In particular, it is important to note that a Procrustes transformation can not change any angles in a group configuration. If four points fall on vertices of a rectangle before Procrustes transformation then they must fall on the vertices of a rectangle after the transformation (and vice versa).

Figure 5 shows the stimulus coordinates (black diamonds) for each pair of stimulus dimensions along with the MDS coordinates (empty circles) after a Procrustes transformation. Note that in every case, the orthogonality of the stimulus dimensions is preserved in the MDS solution. Thus, these results support the hypothesis that the various stimulus dimensions that define the fish stimuli are perceived independently of each other, at least for the five stimulus dimensions varied in Experiment 2.

\section{Discussion}

Experiment 2 provided a variety of reassuring results that strengthen the case for the use of the fish stimuli in cognitive research. First, the stress analysis strongly confirmed that participants attended separately to all five stimulus dimensions that we varied. Thus, at least for the five dimensions we varied, we found no evidence that participants ignored a dimension or that they combined two dimensions into one emergent perceptual dimension. Thus, our results suggest that if researchers create a set of stimuli that vary on the five dimensions manipulated in Experiment 2, then the resulting perceptual representations will also be five dimensional.

Second, Experiment 2 supported the hypothesis that all five dimensions that were varied are perceived independently by participants. Thus, if researchers choose a set of stimuli by factorially combining a set of discrete values on some subset of the dimensions that were varied in Experiment 2, then the orthogonality of the dimensions that were selected should be preserved in the perceptual representations.

\section{General Discussion}

Almost all cognitive research has settled on one of two types of stimuli - either simple artificial stimuli, such as Gabor patches, that vary on only a couple of dimensions (e.g., spatial frequency and orientation) and have a well understood

\footnotetext{
${ }^{1}$ Participants rated similarity from 1 to 7 , so we defined dissimilarity as $8-$ similarity. Note that this standard transformation is linear, which means that the $r^{2}$ for a plot of MDS distances against dissimilarities is the same as the $r^{2}$ for a plot of MDS distances against similarities.
} 

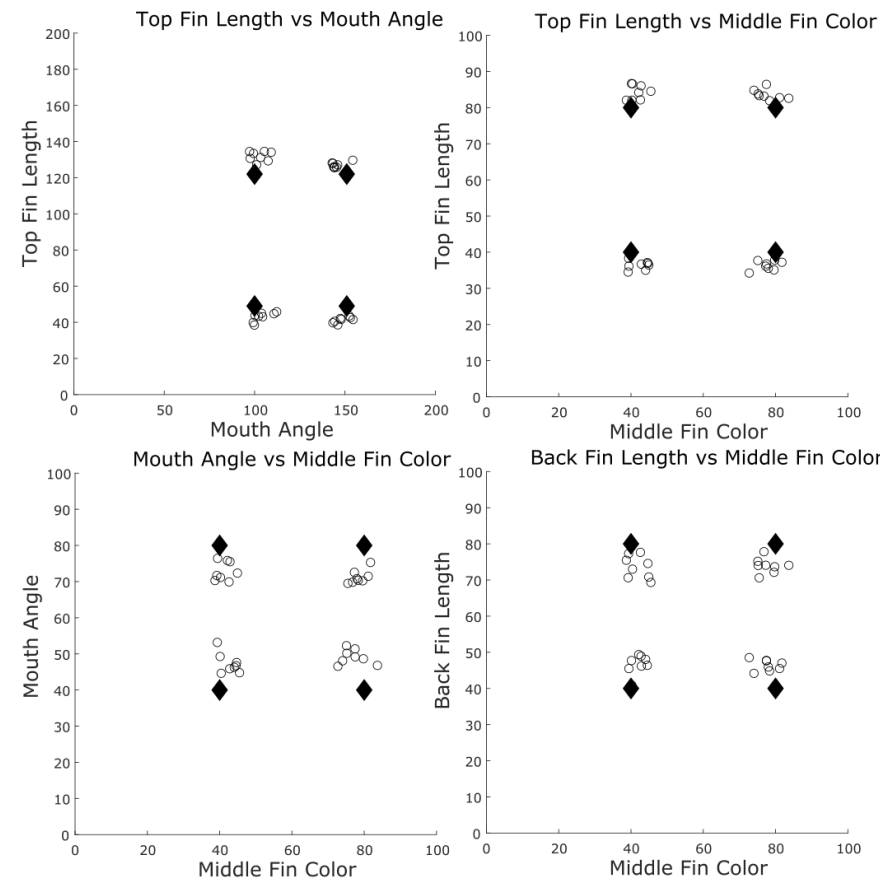

Back Fin Length vs Middle Fin Color
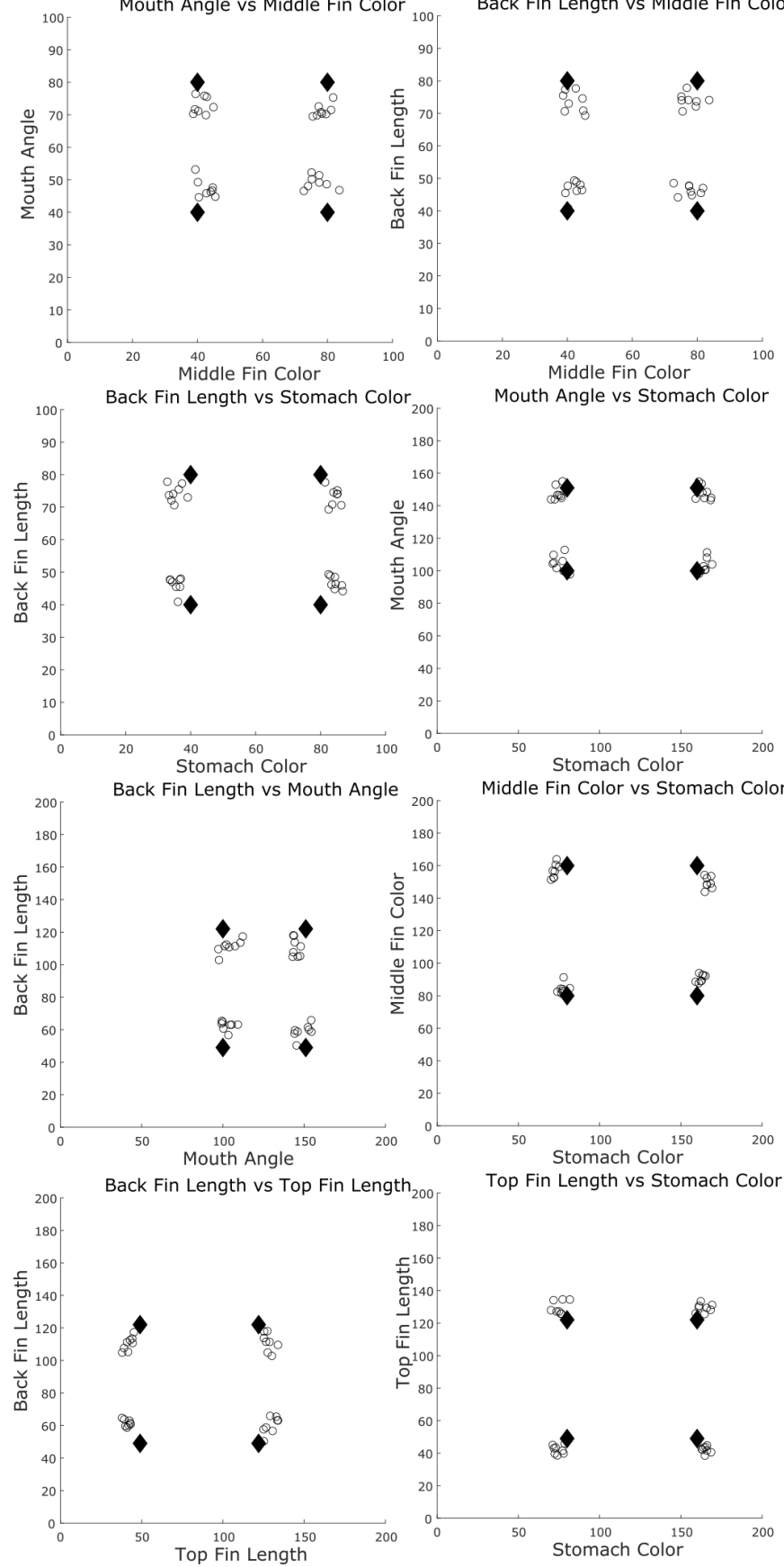

Figure 5. Stimulus coordinates (black diamonds) and MDS points (empty circles) after alignment via Procrustes transformation. perceptual representation, or more complex real-world stimuli (e.g., faces) that vary on an unknown number of dimensions and have a poorly understood perceptual representation. The goal of this research was to bridge this gap by developing a stimulus set of intermediate complexity that is at least reminiscent of real-world stimuli, varies on many dimensions, has spatially separated features to better facilitate feature attention studies, and has a simple and well understood perceptual representation.

Experiment 1 showed how to construct a set of the fish stimuli that lie in a space where the units on each dimension are jnds. First one selects the desired stimulus coordinates of all stimuli in the ensemble where the values on every dimension are jnds. Second, Equation 3 is used to find the length in pixels of all height and width dimensions, and Equation 5 is used to find the angle in degrees of all mouth angles. After constructing the stimuli in this way, Experiment 2 provides reassurance that the spatial configuration of all stimuli in perceptual space will closely match their spatial configuration in physical jnd space. Finally, images of the fish that match the selected stimulus ensemble can be constructed using the Matlab code associated with this article.

In addition to the dimensions investigated in this article, the fish stimuli also have a wealth of other attributes that could be used as features if even higher dimensionality is desired (e.g., distances between fins, eye size, or any of the other constants from Table 1 or Figure 1). Additionally, features such as texture can easily be integrated to increase the dimensionality even further or if users desire to use a specific feature type.

Another advantage of the fish stimuli is that they might be more interesting for participants to interact with than typical low-dimensional artificial stimuli. In our own anecdotal experience, participants have reported less fatigue and more interest after categorizing fish than after categorizing Gabor patches. To make cognitive tasks even more interesting, experimenters might present the experimental task as a game - such as fishing for a specific hypothetical fish species that might require a certain type of lure to catch.

There is a great need within cognitive research for highdimensional stimuli with some real-world properties that are easy to construct and have straight-forward, well-understood perceptual representations. We believe the new fish stimuli described here have promising potential to fill this critical void in the literature.

\section{References}

Ashby, F. G., \& Townsend, J. T. (1986). Varieties of perceptual independence. Psychological Review, 93(2), 154-179.

Jameson, D., \& Hurvich, L. M. (1959). Perceived color and its dependence on focal, surrounding, and preceding stimulus variables. JOSA, 49(9), 890-898. 
Tudusciuc, O., \& Nieder, A. (2010). Comparison of length judgments and the müller-lyer illusion in monkeys and humans. Experimental brain research, 207(3-4), 221-231.

\section{Appendix}

\section{Derivation of Equation 3}

Let $H(X)$ denote the value of $H$ when the number of jnds equals $X$. Note that $H(X)$ is one jnd greater than $H(X-1)$ and the jnd at $H(X-1)$ is $.1 H(X-1)$. Therefore,

$$
H(X)=H(X-1)+.1 H(X-1)=1.1 H(X-1) .
$$

Therefore,

$$
H(2)=1.1 H(1)
$$

and

$$
H(3)=1.1 H(2)=1.1^{2} H(1)
$$

By induction

$$
H(X)=H(1) \times 1.1^{X-1}
$$

Equation 3 follows under the assumption that $H(0)=10$.

\section{Derivation of Equation 5}

Let $\theta(X)$ denote the value of $\theta$ when the number of jnds equals $X$. By the same logic that led to Equation 8, it follows that

$$
\begin{aligned}
\theta(X) & =\theta(X-1)+[.1 \theta(X-1)+2.8] \\
& =1.1 \theta(X-1)+2.8
\end{aligned}
$$

Therefore,

$$
\theta(2)=1.1 \theta(1)+2.8
$$

and

$$
\begin{aligned}
\theta(3) & =1.1 \theta(2)+2.8 \\
& =1.1[1.1 \theta(1)+2.8]+2.8 \\
& =1.1^{2} \theta(1)+2.8(1+1.1) .
\end{aligned}
$$

Similarly,

$$
\begin{aligned}
\theta(4) & =1.1 \theta(3)+2.8 \\
& =1.1\left[1.1^{2} \theta(1)+2.8(1+1.1)\right]+2.8 \\
& =1.1^{3} \theta(1)+2.8\left(1+1.1+1.1^{2}\right) .
\end{aligned}
$$

It follows by induction that

$$
\theta(X)=1.1^{X-1} \theta(1)+2.8 \sum_{i=0}^{X-1} 1.1^{i} .
$$

The sum on the right is a geometric series. By standard rules

$$
\begin{aligned}
\theta(X) & =1.1^{X-1} \theta(1)+2.8\left(\frac{1-1.1^{X}}{1-1.1}\right) \\
& =1.1^{X-1} \theta(1)+28 \times 1.1^{X}-28 \\
& =\frac{1.1}{1.1}\left[1.1^{X-1} \theta(1)\right]+28 \times 1.1^{X}-28 \\
& =\left[\frac{\theta(1)}{1.1}+28\right] 1.1^{X}-28 .
\end{aligned}
$$

If we choose $\theta(1)=11$ then Equation 17 reduces to Equation 5 . 


\section{Acknowledgments}

This material is based upon work supported by the $\mathrm{Na}$ tional Science Foundation Graduate Research Fellowship un- der Grant No. 1650114 and by National Institutes of Health grant 2R01MH063760. Thanks to Mikayla Davis for her assistance with data collection. 